Charles L. Sprung

Thomas Woodcock

Peter Sjokvist

Bara Ricou

Hans-Henrik Bulow

Anne Lippert

Paulo Maia

Simon Cohen

Mario Baras

Seppo Hovilehto

Didier Ledoux

Dermot Phelan

Elisabet Wennberg

Wolfgang Schobersberger

\section{Reasons, considerations, difficulties and documentation of end-of-life decisions in European intensive care units: the ETHICUS Study}

Received: 6 November 2005

Accepted: 11 October 2007

Published online: 9 November 2007

(c) Springer-Verlag 2007

For the ETHICUS Study Group

P. Sjokvist died during the preparation of this manuscript.

\section{L. Sprung (}

Hadassah Hebrew University Medical

Center, General Intensive Care Unit,

Department of Anesthesiology and Critical

Care Medicine

P.O. Box 12000, 91120 Jerusalem, , Israel

e-mail: sprung@cc.huji.ac.il

T. Woodcock

Southampton University Hospitals NHS

Trust, Critical Care Directorate,

Southampton, UK

\section{P. Sjokvist}

Orebro and Huddinge University Hospital,

Department of Anesthesiology,

Stockholm, Sweden

\section{B. Ricou}

Hopital Cantonal Universitaire de Geneve,

Department of Anesthesiology,

Geneva, Switzerland

\section{H.-H. Bulow}

University Hospital of Glostrup,

Department of Anesthesiology,

Glostrup, Denmark

\section{A. Lippert}

Herlev University Hospital, Department of Anesthesiology,

Herlev, Denmark
P. Maia

Hospital Geral Santo Antonio, Department of Intensive Care,

Porto, Portugal

\section{S. Cohen}

University College of London, Department of Medicine,

London, UK

\section{Baras}

Hadassah Hebrew University Medical

Center, The Hebrew University - Hadassah

School of Public Health,

Jerusalem, Israel

S. Hovilehto

South Karelia Central Hospital, Department of Anesthesiology,

Lappeenranta, Finland

\section{Ledoux}

University of Liege, Department of Anesthesiology and Intensive Care

Medicine,

Liege, Belgium

D. Phelan

Mater Hospital, University College,

Department of Intensive Care,

Dublin, Ireland

\section{E. Wennberg}

Sahlgrenska University Hospital,

Department of Anesthesia and Intensive

Care,

Gothenburg, Sweden

\section{W. Schobersberger}

University Hospital Innsbruck, Division for General and Surgical Intensive Care

Medicine,

Innsbruck, Austria
Abstract Objective: To evaluate physicians' reasoning, considerations and possible difficulties in end-of-life decision-making for patients in European intensive care units (ICUs). Design: A prospective observational study. Setting: Thirty-seven ICUs in 17 European countries. Patients and participants: A total of 3,086 patients for whom an end-of-life decision was taken between January 1999 and June 2000. The dataset excludes patients who died after attempts at cardiopulmonary resuscitation and brain-dead patients. Measurements and results: Physicians indicated which of a pre-determined set of reasons for, considerations in, and difficulties with end-of-life decision-making was germane in each case as it arose. Overall, $2,134(69 \%)$ of the decisions were documented in the medical record, with inter-regional differences in documentation practice. Primary reasons given by physicians for the decision mostly concerned the patient's medical condition (79\%), especially unresponsive to therapy (46\%), while chronic disease (12\%), quality of life $(4 \%)$, age $(2 \%)$ and patient or family request $(2 \%)$ were infrequent. Good medical practice $(66 \%)$ and best interests $(29 \%)$ were the commonest primary considerations reported, 
while resource allocation issues such as cost effectiveness (1\%) and need for an ICU bed $(0 \%)$ were uncommon. Living wills were considered in only $1 \%$ of cases. Physicians in central Europe reported no significant difficulty in $81 \%$ of cases, while in northern and southern regions there was no difficulty in 92-93\% of cases. Conclusions: European ICU physicians do not experience difficulties with end-of-life decisions in most cases. Allocation of limited resources is a minor consideration and autonomous choices by patient or family remain un- usual. Inter-regional differences were found.

Keywords Withholding treatment . Physician's role · Life-support care · Intensive care units - Europe . Decision-making $\cdot$ Reasons $\cdot$ Difficulties $\cdot$ Considerations

\section{Introduction}

Descriptions of practices of forgoing life support in intensive care units (ICUs) date from the 1970s [1]. During the past decade treatment limitations have been reported to precede most deaths [2-8]. In the USA, Prendergast documented a major change in practice with more limitations (especially withdrawing life support) between the late 1980s and early 1990s and reported that physicians experienced few specific difficulties with this change [8]. Generally, end-of-life decisions are described as difficult [9-12].

These judgments demand that decision-makers balance important ethical and legal principles such as the sanctity of life, the right of a patient to determine how he/she shall be treated, and the expectation that a doctor's first consideration will be the welfare or best interests of the patient. However, ICUs have only a limited capacity and resource allocation considerations have to be taken into account. Intensive care practices may involve disputes between patients, patients' representatives, and their treating professionals about the way vital decisions are made and raise important social, ethical, and legal questions [13]. In a French study, decisions to forgo life-sustaining treatments in the ICU were found to be independent of objective measures such as severity of illness score and comorbidities and strongly associated with death [14]. Previously published reports from the ETHICUS database have addressed the ways patients die [7], involvement of patients and families [15], physician religion [16], regional culture $[17,18]$, and the role of nurses in end-of-life decisions [19]. However, little information is available regarding the values physicians use to make end-of-life decisions [20]. This ETHICUS study evaluated physician documentation and reasons, considerations and difficulties in end-of-life decision-making in ICUs.

\section{Methods}

ETHICUS was a prospective study of end-of-life practices and decisions in consecutive patients who died or were subject to any limitation of life-saving interventions in 37 ICUs in 17 European countries from 20 January 1999 until 30 June 2000. Patients were followed until discharge, death, or 2 months from the decision to limit therapy. This paper presents data of patients who had life support withheld, withdrawn or the dying process actively shortened [7]. Patients in whom death was preceded by unsuccessful cardiopulmonary resuscitation (CPR) or who were brain dead were not examined in this study. Patients were classified as 'withhold' only if that was the sole limitation made, 'withdraw' included patients for whom both withholding and withdrawing limitations were made, and 'shortening of the dying process' (SDP) included patients with withholding or withdrawing and SDP decisions. Countries were divided into three regions (northern, central and southern) based on geographic location prior to data analysis as different practices based on geography have been suggested $[7,21]$.

Following institutional ethics approval and a waiver of informed consent, data were collected in each institution as events occurred. Data on documentation collected included whether there was a written do-not-resuscitate (DNR) order and whether the end-of-life decision was documented in the medical record. Reporting physicians were ICU clinicians responsible for patients who were asked which of several pre-defined "primary reasons" "primary considerations" and "primary difficulties" applied in decision-making. In the instructions to the question of 'difficulty' only those decisions that included difficulties "out of the ordinary" were to be reported. The reason "unresponsive to therapy" was defined as either a shock patient who does not increase blood pressure to vasopressors or a hypoxemic patient who does not increase arterial oxygen tension to increasing inspired oxygen fraction and end-expiratory pressure.

\section{Statistical methods}

The incidence of reasons, considerations and difficulties arising are described in total numbers and as a percentage of the cases. Times are reported as medians and inter-quartile range (IQR). For inter-regional comparisons of times the Kruskal-Wallis test was used and for dichotomies the Mann-Whitney test. For all other items, a chi-square test was used. To account for multiple com- 
parisons the $p$ values were adjusted by Holm's method. A test was considered significant if $p<0.05$.

\section{Results}

During the ETHICUS study, 31,417 patients were admitted to ICUs in 37 centers located in 17 countries over 13.5 months. Of the 31,417 patients, 4,280 died or had limitations of life-sustaining treatments. Of these 4,280 patients, 32 were excluded on grounds of age or the absence of end-of-life information. Thus, 4,248 patients died or had end-of-life decisions. After excluding patients who were categorized as brain dead [330 (8\%)] or died after unsuccessful CPR [832 (30\%)], 3,086 patients remain in whom life support was withheld, withdrawn, or SDP was decided upon; these make up the present study. Decisions to withhold life-sustaining therapy were made in 1594 (52\%) cases, to withdraw it in 1398 (45\%) cases, and to shorten the dying process in 94 (3\%) cases. A written order for DNR was made in $1,991(65 \%)$ of 3,081 cases and all end-of-life decisions were documented in the medical record in 2,134 (69\%) of 3,079 cases. The proportion of cases with casenote-documented decisions was lower in the southern region (29\% DNR, 34\% all decisions) than in the central (78\% DNR, 77\% all decisions) and northern (79\% DNR, 88\% all decisions) regions $(p<0.001)$.

The primary reasons for the end-of-life decisions according to region are presented in Table 1. The most frequently cited reason, "unresponsive to therapy" (46\%), was given more often in the southern region $(57 \%)$ than in the central $(38 \%)$ or northern $(45 \%)$ regions $(p<0.001)$. The time from ICU admission to the first end-of-life decision was longer for patients with unresponsive to therapy as a reason to limit treatment $(8.6+18.3$ days $)$ than in those with other reasons $(7.4+21.3$ days; $p<0.001)$. Other reasons included neurologic grounds $(20 \%)$, chronic disease $(12 \%)$, and multi-organ failure (10\%). Poor quality of life $(4 \%)$, patient or family request $(2 \%)$ or age $(2 \%)$ were infrequent reasons. Age was less often cited in the northern region than in the central or southern regions $(p<0.001)$. Figure 1 illustrates the proportion of cases in which "unresponsive to therapy" was the primary reason in each ICU.

"Good medical practice" (66\%) and "best interests of the patient" (29\%) were the leading considerations in forgoing life-sustaining treatments in all three European regions (Table 2). Considerations of autonomous decisions by the patient, living wills, ICU bed requirements, costs, or legal concerns were infrequent. In only $25 \%$ of patients

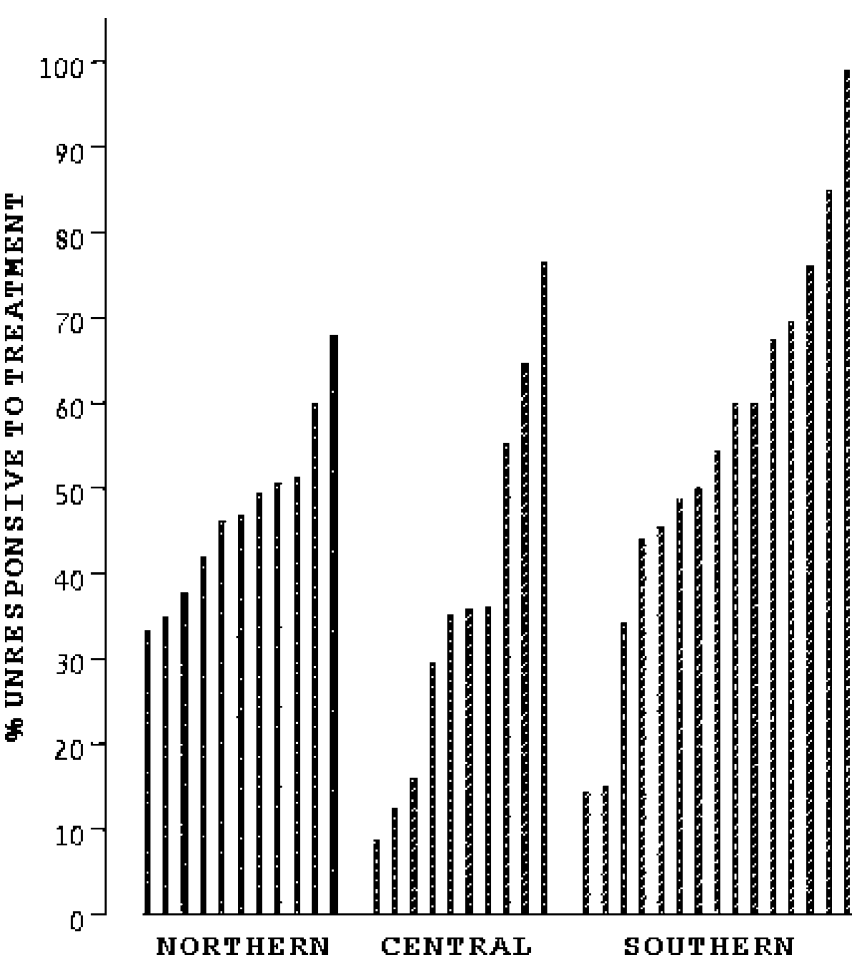

Fig. 1 Unresponsive to therapy reason for end-of-life decision in each ICU according to region
Table 1 Primary reasons for end-of-life decision by region

\begin{tabular}{lcccc}
\hline & Northern & Central & Southern & \multicolumn{1}{l}{ Total } \\
\hline Unresponsive to maximal therapy* & $582(45.0)$ & $336(37.6)$ & $507(57.4)$ & $1,425(46.4)$ \\
Neurologic & $262(20.2)$ & $206(23.0)$ & $147(16.6)$ & $615(20.0)$ \\
Chronic disease & $200(15.5)$ & $106(11.9)$ & $73(8.3)$ & $379(12.3)$ \\
Multi-organ failure & $126(9.7)$ & $97(10.9)$ & $72(8.2)$ & $295(9.6)$ \\
Poor quality of life & $27(2.1)$ & $69(7.7)$ & $30(3.4)$ & $126(4.1)$ \\
Sepsis/septic shock & $45(3.5)$ & $39(4.4)$ & $20(2.3)$ & $104(3.4)$ \\
Patient/family request & $42(3.2)$ & $11(1.2)$ & $12(1.4)$ & $65(2.1)$ \\
Age & $4(0.3)$ & $23(2.6)$ & $19(2.2)$ & $46(1.5)$ \\
Other & $6(0.5)$ & $7(0.8)$ & $3(0.3)$ & $16(0.5)$ \\
Total & $1,294(100)$ & $894(100)$ & $883(100)$ & $3,071(100)$ \\
\hline
\end{tabular}

Number (percent)

* Shock patient who did not react with increased blood pressure to vasopressors or a hypoxemic patient who did not react with increased $\mathrm{PaO}_{2}$ to increasing $\mathrm{FIO}_{2}$ and PEEP. 
Table 2 Primary considerations taken in end-of-life decisions by region

\begin{tabular}{|c|c|c|c|c|}
\hline & Northern & Central & Southern & Total \\
\hline Good medical practice & $855(65.7)$ & $553(61.6)$ & $617(71.1)$ & $2,025(66.0)$ \\
\hline Best interest of patient & $389(29.9)$ & 284 (31.6) & $201(23.2)$ & $874(28.5)$ \\
\hline Autonomous decision of patient & $35(2.7)$ & $16(1.8)$ & $11(1.3)$ & $62(2.0)$ \\
\hline Cost effectiveness & $2(0.2)$ & $14(1.6)$ & $12(1.4)$ & $28(0.9)$ \\
\hline Living will & $3(0.2)$ & $13(1.4)$ & $12(1.4)$ & $28(0.9)$ \\
\hline Social and family pressures & $6(0.5)$ & $2(0.2)$ & $9(1.0)$ & $17(0.6)$ \\
\hline Legal concerns & $2(0.2)$ & $2(0.2)$ & $2(0.2)$ & $6(0.2)$ \\
\hline Religious principles & $2(0.2)$ & - & - & $2(0.1)$ \\
\hline Need for ICU bed & - & - & $1(0.1)$ & $1(0.0)$ \\
\hline Other & $7(0.5)$ & $14(1.6)$ & $3(0.3)$ & $24(0.8)$ \\
\hline Total & $1,301(100)$ & $898(100)$ & $868(100)$ & $3,067(100)$ \\
\hline
\end{tabular}

Number (percent) with a living will was the living will rated as the primary consideration.

Table 3 demonstrates the primary difficulty in making end-of-life decisions by region. The deciding physician perceived no difficulty in $89 \%$ of the cases, with physicians in the central region least often reporting no difficulty $(p<0.001)$. The time from ICU admission to the first end-of-life decision was shorter for patients with no difficulties in decisions to limit therapy $(7.7+18.6$ days) than in those with any difficulty $(10.4+15.0$ days; $p<0.001)$. Figure 2 illustrates the "no difficulty" reports from each ICU.

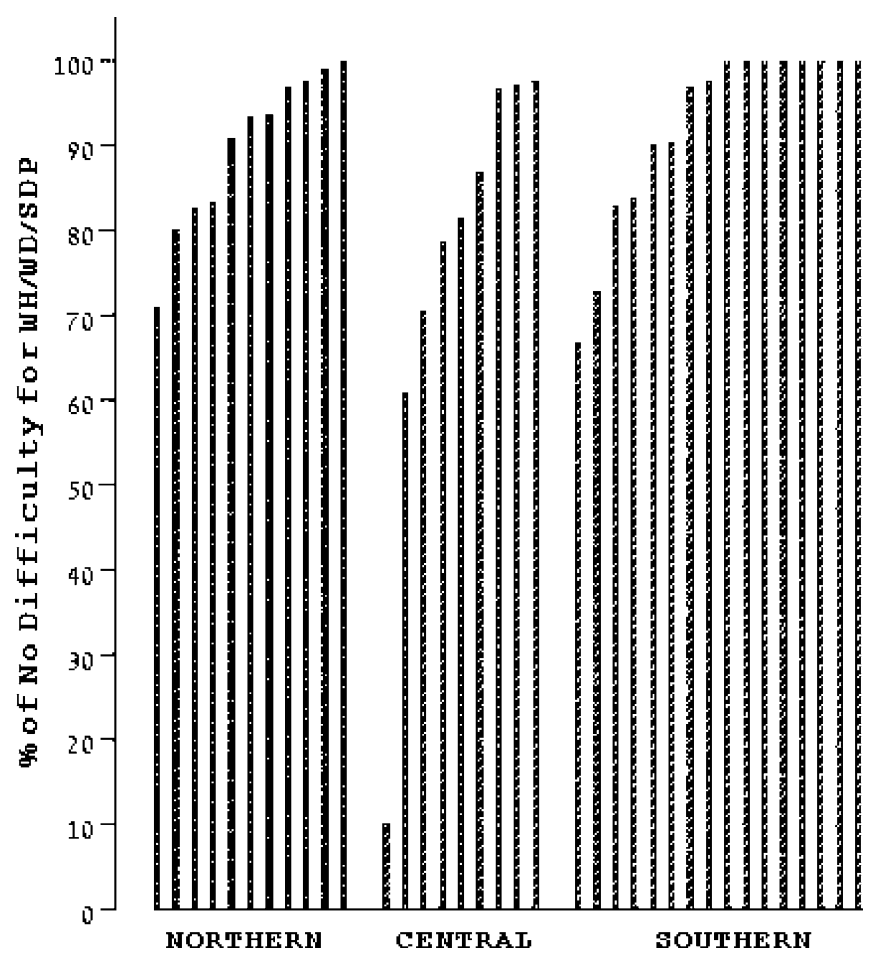

Fig. 2 No difficulty in end-of-life decision in each ICU according to region
Table 3 Primary difficulty in making end-of-life decisions by region

\begin{tabular}{lcccc}
\hline & Northern & Central & \multicolumn{1}{c}{ Southern } & \multicolumn{1}{l}{ Total } \\
\hline None & $1,198(92.2)$ & $727(81.0)$ & $820(93.1)$ & $2,745(89.2)$ \\
Medical & $59(4.5)$ & $63(7.0)$ & $27(3.1)$ & $149(4.8)$ \\
Ethical & $15(1.2)$ & $54(6.0)$ & $17(1.9)$ & $86(2.8)$ \\
Legal & $12(0.9)$ & $38(4.2)$ & $13(1.5)$ & $63(2.0)$ \\
Religious & $4(0.3)$ & $4(0.4)$ & $2(0.2)$ & $10(0.3)$ \\
Other & $12(0.9)$ & $12(1.3)$ & $2(0.2)$ & $26(0.8)$ \\
Total & $1,300(100)$ & $898(100)$ & $881(100)$ & $3,079(100)$ \\
\hline
\end{tabular}

Number (percent)

\section{Discussion}

This is the first large, international prospective study evaluating physician documentation and reasons for end-of-life decision-making, considerations taken in these decisions, and the difficulties physicians experience in making these choices. Substantial regional variability in the limitations and the dying process in European ICUs were previously reported by the ETHICUS Group [7]. It is therefore important to examine the reasons physicians gave for their decisions as an important step towards understanding these inter-regional differences in practice. The present study demonstrated that physicians from the southern region compared to the northern and central regions more commonly gave as a reason for limiting therapy "unresponsive to therapy" and less commonly chronic disease, less commonly gave the best interests of the patient as a consideration, and less commonly documented their decisions. This conservative, paternalistic pattern in the south corresponds to the previous ETHICUS findings in the south of more CPR, less limitations and less communication with patients and families $[7,15]$.

Several previous studies described the approaches, attitudes, and preferences of intensivists in limiting life support [21-26]. Some observational studies have focused both on the motivation for end-of-life decisions in actual cases [27-31] and the pathophysiological issues physicians focus on [32-34]. 


\section{Reasons for limitation decisions}

In this study the commonest primary reason for the endof-life decision was unresponsiveness to therapy. In the southern region, more than half of the limitation decisions were primarily justified by unresponsiveness to therapy. The southern region also reported the highest proportion of deaths with no limitation decisions [7]. Physician perseverance until all hope of patient survival has gone appears strongest in southern European ICUs. Predicting individual outcomes from critical illnesses remains an imprecise science, but an end-of-life decision can more easily be justified when the physician concludes that the patient is unresponsive to treatment or has severe neurological injury.

Poor quality of life was rarely cited by physicians as a reason for limitation. This finding is similar to a Canadian observational study in which poor quality of life was mentioned, but less often than poor prognosis [28]. In contrast, quality of life reasons were the most frequent ones cited in a British single-center study [30]. Central region physicians reported poor quality of life as the reason more often than physicians from the northern or southern regions. This is a controversial reason for denying life-sustaining treatment, and the fact that central region physicians also reported more ethical difficulties in decision-making than their colleagues suggests that ethical sensibilities may be stronger there. It can be argued that quality of life is not a value judgment that doctors are especially qualified to make and that people with disabilities may be especially vulnerable to negative perceptions by able-bodied carers about their quality of life. Comparison with other published studies is complicated by the fact that other researchers have generally classified all physician decisions broadly into futility versus quality categories, whereas the ETHICUS questionnaire gave physicians a wider choice of reasons.

\section{Considerations for limitation decisions}

Unsurprisingly, physicians of all regions placed greatest reliance on their interpretation of good medical practice as their primary consideration in decision-making. This corresponds with the suggestion that end-of-life decisions are mostly considered to be professional or medical judgments [25] and that the reasoning of physicians focuses primarily on medical issues [30]. Institutional, national, and international guidelines on what constitutes good medical practice are therefore likely to be powerful tools to bring about changes in practices.

Patient or family request was a reason for limitation in $2 \%$ of cases and autonomous decision of the patient or living will a consideration in $2 \%$ and $1 \%$, respectively. Patients and families are not adequately encouraged to take more than a peripheral role in end-of-life decisionmaking [35], and physicians excessively dominate the decision-making process [36] in a paternalistic manner. The data presented here tend to support this view but it must be noted that other studies have suggested that more family involvement occurs in most [9, 28, 30] or some [4, 25] end-of-life decisions. Expecting or even requiring autonomous decisions from patients or families in critical care practice presents many difficulties [37].

\section{Difficulties in limitation decisions}

Decisions concerning limitation of life support for patients in ICUs are presumed to be difficult for all parties concerned. Although previous studies demonstrated that end-of-life decisions were difficult in up to $72 \%$ of discussions [12], European physicians reported experiencing significant difficulties in only $11 \%$ of end-of-life decisions. Even in the central region, in which the physicians were most inclined to see the decisions as difficult, more than $80 \%$ were not considered difficult.

Nevertheless, the time from ICU admission to the first end-of-life decision was shorter for patients with no difficulties in decisions to limit therapy than for those with any difficulty. Though the ETHICUS database shows an association between end-of-life practices across Europe and physician and patient religion [7, 16], this report shows that the physicians themselves do not perceive religion to be a consideration or to present difficulties.

Non-physician observers and other participants in the decision-making process may see more difficulties than physicians report. In an international study, nurses were found to experience more 'discomfort' with life-support plans than physicians [34]. In a French survey, the majority of physicians believed that their end-of-life decisionmaking processes were satisfactory, while only a minority of nurses reported satisfaction [37]. In Canada, wide variations were found in the satisfaction expressed by nonphysician health care professionals about physician-led decisions [38].

It may be that physicians do not describe decisions as difficult because they wait until the prognosis is relatively obvious to all before justifying the decision on medical grounds. Patients with 'unresponsive to therapy' as a reason to limit treatment had longer times from ICU admission to the first end-of-life decision. Prognostic uncertainty leads physicians to pursue aggressive therapy [39], and waiting until death appears certain causes less difficulties in making decisions. Doctors give medical reasons for forgoing life-sustaining treatments which may be part of an excessively paternalistic approach to end-of-life decisionmaking.

It must be acknowledged that the phrasing of the instructions to physicians stated that only cases with 'more than ordinary' difficulties should be marked as 'difficult' and so lesser difficulties were not reported. Nonetheless, this study suggests that end-of-life decisions have become 
part of everyday life in European ICUs and are treated by physicians as mainly medical judgments that in most cases are not more than ordinarily difficult. It could be surmised that difficulties which consumed additional time and resources would likely be categorized as more than ordinarily difficult. Inquiries about sequelae of reported difficulties were not made, and some ICUs may have expended a significant amount of time on the resolution of the small number of difficulties.

\section{Documentation of limitation decisions}

Medical record documentation of end-of-life decisionmaking processes would be expected in all cases, but in the southern region only approximately one third of the cases had written documentation, while the proportion was nearer to two thirds in the central and northern regions. A previous study documented documentation in approximately half the patients [30], and a questionnaire survey of European intensive care physicians also revealed a south-north difference regarding the use of written DNR orders [21].

The major strengths of the present study are the direct reporting of actions rather than theoretical responses to a questionnaire, the prospective enrollment of a large number of consecutive patients from 37 ICUs in 17 countries, and the evaluation of all treatment limitations and deaths in all admitted patients. Anonymity and contemporaneous documentation were used to capture frank and accurate reporting of a very sensitive and potentially emotive process. There are some weaknesses in the study. The patients studied may not be a representative sample of the ICU population of each European country. Since there were only one to four centers participating in each country, it was recognized a priori that the participating ICUs could not claim to represent the practices of all ICUs in that coun- try. Therefore, the larger groupings of regions were evaluated. In addition, participant physicians, by their special interest in ethical issues, may not necessarily share the attitudes of a random selection of ICU doctors. The patient's severity of illness at the time of decision-making was not recorded and a different case-mix of patients in different regions could account for some of the observed differences. Different phrasing of questions and instructions may have given different answers. Intensive care professionals are expected to engage in a shared approach to endof-life decision-making involving the caregiver team and patient surrogates, showing respect for patient autonomy and the intention to honor decisions to decline unwanted treatments. The ETHICUS study has shown that European practices, and indeed European patients, have some way to travel towards achieving these objectives. The results of this study suggest that there is room for greater engagement with patients and their families to promote patient autonomy in the end-of-life decision-making process. European intensive care physicians are very comfortable taking a leading role in this process and they report only a small number of cases in which they experience difficulty with the biggest category of difficulties relates to medical issues. It is important that future studies look beyond the role of physicians. If patients, their families, and other professionals are to be encouraged to participate more actively in the process of end-of-life decision-making, it will be helpful for physicians and policy-makers to understand what other health care providers believe and most importantly, how patients and families view these end-of-life issues.

Acknowledgements. This study was funded by the European Concerted Action Project and by the European Commission (Contract PL 963733), the Chief Scientists' Office of the Ministry of Health, Israel (grant 4226), and the OFES Switzerland/Biomed (grant 980271). The study also received funding from the European Society of Intensive Care Medicine and the Walter F and Alice Gorham Foundation Inc.

\section{References}

1. Sprung CL (1990) Changing attitudes and practices in forgoing life-sustaining treatments. JAMA 263:2211-2215

2. Prendergast TJ, Claessens MT, Luce JM (1998) A national survey of end-of-life care for critically ill patients. Am J Respir Crit Care Med 158:1163-1167

3. Ferrand $E$, Robert $R$, Ingrand $P$, Lemaire F (2001) Withholding and withdrawal of life support in intensivecare units in France: a prospective survey. French LATAREA Group. Lancet 357:9-14
4. Esteban A, Gordo F, Solsona JF, Alia I, Caballero J, Bouza C, Alcala-Zamora J, Cook DJ, Sanchez JM, Abizanda R, Miro G, Fernandez Del Cabo MJ, de Miguel E, Santos JA, Balerdi B (2001) Withdrawing and withholding life support in the intensive care unit: a Spanish prospective multi-centre observational study. Intensive Care Med 27:1744-1749

5. Sjokvist P, Sundin PO, Berggren L (1998) Limiting life support. Experiences with a special protocol. Acta Anaesthesiol Scand 42:232-237
6. Keenan SP, Busche KD, Chen LM, Esmail R, Inman KJ, Sibbald WJ (1998) Withdrawal and withholding of life support in the intensive care unit: a comparison of teaching and community hospitals. The Southwestern Ontario Critical Care Research Network. Crit Care Med 26:245-251

7. Sprung CL, Cohen SL, Sjokvist P, Baras M, Bulow HH, Hovilehto S, Ledoux D, Lippert A, Maia P, Phelan D, Schobersberger W, Wennberg E, Woodcock T (2003) End-of-life practices in European intensive care units: the Ethicus Study. JAMA 290:790-797 
8. Prendergast TJ, Luce JM (1997) Increasing incidence of withholding and withdrawal of life support from the critically ill. Am J Respir Crit Care Med 155:15-20

9. The SUPPORT Principal Investigators (1995) A controlled trial to improve care for seriously ill hospitalized patients. The study to understand prognoses and preferences for outcomes and risks of treatments (SUPPORT). JAMA 274:1591-1598

10. Soderberg A, Norberg A (1993) Intensive care: situations of ethical difficulty. J Adv Nurs 18:2008-2014

11. Fetters MD, Churchill L, Danis M (2001) Conflict resolution at the end of life. Crit Care Med 29:921-925

12. Sharma BR (2004) Withholding and withdrawing of life support: a medicolegal dilemma. Am J Forensic Med Pathol 25:150-155

13. Schaller C, Kessler M (2006) On the difficulty of neurosurgical end of life decisions. J Med Ethics 32:65-69

14. Azoulay E, Pochard F, GarrousteOrgeas M, Moreau D, Montesino L, Adrie C, de Lassence A, Cohen Y, Timsit JF (2003) Decisions to forgo life-sustaining therapy in ICU patients independently predict hospital death. Intensive Care Med 29:1895-1901

15. Cohen S, Sprung CL, Sjokvist P, Lippert A, Ricou B, Baras M, Hovilehto S, Maia P, Phelan D, Reinhart K, Werdan K, Bulow HH, Woodcock T (2005) Communication of end of life decisions in European intensive care units - The Ethicus Study. Intensive Care Med 31:1215-1221

16. Sprung CL, Maia P, Bulow HH, Ricou B, Armaganidis A, Baras M, Wennberg E, Reinhart K, Cohen SL, Fries DR, Nakos G, Thijs LG (2007) The importance of religious affiliation and culture on end-of-life decisions in European intensive care units. Intensive Care Med 33:1732-1739

17. Ganz FD, Benbenishty J, Hersch M, Fischer A, Gurman G, Sprung CL (2006) The impact of regional culture on intensive care end of life decision making: an Israeli perspective from the ETHICUS study. J Med Ethics 32:196-199
18. Collins N, Phelan D, Marsh B, Sprung CL (2006) End-of-life care in the intensive care unit: the Irish Ethicus data. Crit Care Resuscitation 8:315-320

19. Benbenishty J, Ganz FD, Lippert A, Bulow HH, Wennberg E, Henderson B, Svantesson M, Baras M, Phelan D, Maia P, Sprung CL (2006) Nurse involvement in end-of-life decision making: the ETHICUS study. Intensive Care Med 32:129-132

20. Rubenfeld GD, Curtis JR (2001) End-of-life care in the intensive care unit: a research agenda. Crit Care Med 29:2001-2006

21. Vincent JL (1999) Forgoing life support in western European intensive care units: the results of an ethical questionnaire. Crit Care Med 27:1626-1633

22. Cook DJ, Guyatt GH, Jaeschke R, Reeve J, Spanier A, King D, Molloy DW, Willan A, Streiner DL (1995) Determinants in Canadian health care workers of the decision to withdraw life support from the critically ill. Canadian Critical Care Trials Group. JAMA 273:703-708

23. Cook D, Rocker G, Marshall J, Sjokvist P, Dodek P, Griffith L, Freitag A, Varon J, Bradley C, Levy M, Finfer S, Hamielec C, McMullin J, Weaver B, Walter S, Guyatt G (2003) Withdrawal of mechanical ventilation in anticipation of death in the intensive care unit. N Engl J Med 349:1123-1132

24. Sjokvist P, Berggren L, Cook DJ (1999) Attitudes of Swedish physicians and nurses towards the use of life-sustaining treatment. Acta Anaesthesiol Scand 43:167-172

25. Yap HY, Joynt GM, Gomersall CD (2004) Ethical attitudes of intensive care physicians in Hong Kong: questionnaire survey. Hong Kong Med J 10:244-250

26. Prendergast TJ (1997) Resolving conflicts surrounding end-of-life care. New Horiz 5:62-71

27. Keenan SP, Busche KD, Chen LM, McCarthy L, Inman KJ, Sibbald WJ (1997) A retrospective review of a large cohort of patients undergoing the process of withholding or withdrawal of life support. Crit Care Med 25:1324-1331

28. Wood GG, Martin E (1995) Withholding and withdrawing life-sustaining therapy in a Canadian intensive care unit. Can J Anaesth 42:186-191
29. Manara AR, Pittman JA, Braddon FE (1998) Reasons for withdrawing treatment in patients receiving intensive care. Anaesthesia 53:523-528

30. Melltorp G, Nilstun T (1996) Decisions to forgo life-sustaining treatment and the duty of documentation. Intensive Care Med 22:1015-1019

31. Eidelman LA, Jakobson DJ, Pizov R, Geber D, Leibovitz L, Sprung CL (1998) Forgoing life-sustaining treatment in an Israeli ICU. Intensive Care Med 24:162-166

32. Koch KA, Rodeffer HD, Wears RL (1994) Changing patterns of terminal care management in an intensive care unit. Crit Care Med 22:233-243

33. Griffith L, Cook D, Hanna S, Rocker G, Sjokvist P (2004) Clinician disconfort with life support plans for mechanically ventilated patients. Intensive care Med 30:1783-1790

34. Sinuff T, Cook DJ, Rocker GM, Griffith LE, Walter SD, Fisher MM, Dodek PM, Sjokvist P, McDonald E, Marshall JC, Kraus PA, Levy MM, Lazar NM, Guyatt GH (2004) DNR directives are established early in mechanically ventilated intensive care unit patients. Can J Anaesth 51:1034-1041

35. Reckling JB (1997) Who plays what role in decisions about withholding and withdrawing life-sustaining treatment. J Clin Ethics 8:39-45

36. Meyers C (2004) Cruel choices: autonomy and critical care decision-making. Bioethics 18:104-119

37. Ferrand E, Lemaire F, Regnier B, Kuteifan K, Badet M, Asfar P, Jaber S, Chagnon JL, Renault A, Robert R, Pochard F, Herve C, Brun-Buisson C, Duvaldestin P (2003) Discrepancies between perceptions by physicians and nursing staff of intensive care unit end-of-life decisions. Am J Respir Crit Care Med 167:1310-1315

38. Keenan SP, Mawdsley C, Plotkin D, Sibbald WJ (2003) Interhospital variability in satisfaction with withdrawal of life support: room for improvement. Crit Care Med 31:626-631

39. Hanson LC, Tulsky JA, Danis M (1997) Can clinical interventions change care at the end of life? Ann Intern Med 126:381-388 